\title{
Sweet's Syndrome: a case report
}

\author{
${ }^{1}$ Kayastha BMM, ${ }^{2}$ Lama L, ${ }^{2}$ Shrestha $\mathrm{P},{ }^{2}$ Shrestha $\mathrm{R},{ }^{2}$ Karki A \\ ${ }^{1}$ Associate Professor and Senior Consultant Dermatologist \\ ${ }^{2}$ Residents \\ Department of Dermatology and STD \\ National Academy of Medical Sciences (NAMS), \\ Bir Hospital, Kathmandu, Nepal \\ Correspondence: bmkayastha@gmail.com
}

\begin{abstract}
Sweet's syndrome is a disorder characterized by fever and painful skin lesions. The condition starts suddenly with the appearance of red, slightly raised tender plaques, usually on the back, arms, face or neck. Women are most at risk of Sweet's syndrome, predominantly between 30-50 years of age who have recently had an upper respiratory tract infection. Here we present a 70 years old lady who came with fever and tender erythematous plaques on trunk and limbs. On investigation, leucocytosis with raised ESR was found and the skin biopsy was consistent with Sweet's syndrome. There was dramatic improvement with systemic corticosteroid.
\end{abstract}

\section{Introduction}

Sweet's syndrome was first described by Robert Douglas Sweet in 1964 as acute febrile neutrophilic dermatosis which primarily affects adult females. It is characterized by fever, peripheral leucocytosis, acute onset of typical skin lesions and histological finding of dense neutrophilic infiltrate. Patients usually have associated systemic findings. Most commonly there is fever (in 50-80\%); arthralgia, myalgia, and arthritis may also be present. Cutaneous lesions consists of erythematous, violaceous, tender papules or nodules that often coalesce to form irregular plaques typically involving the arms, upper trunk, face and extremities.

Histological features consist of dense predominantly neutrophilic infiltrate located in the superficial dermis and prominent papillary dermal oedema. Infiltrate may also contain lymphocytes, eosinophil and histiocytes. The infiltrate may be perivascular or may have band like distribution.

\section{Case report}

A 70years old housewife came to the Dermatology OPD of Bir Hospital on 064/11/08(20 $0^{\text {th }}$ Feb.2008) with 8 days' history of painful skin lesions over the trunk which later progressed to involve the extremities. She reported that she had feverish feeling for 3 weeks prior to presentation. She also reported that she was suffering arthralgia and generalized body ache for 7 days. For this problem she was prescribed a local steroid and non- steroidal anti inflammatory drugs (NSAIDs) with out any improvement. There was no significant past medical or surgical history.

On examination, the patient was febrile and ill looking. There were tender dusky oedematous and erythematous urticarial plaques over the trunk especially on the back as well as both upper and lower limbs. There were few target-like lesions on both the legs. Palms were also involved, but oral and genital mucosae were free.

Investigations: TLC-14,000/cumm; DLC- N79, L19, M2; ESR- $22 \mathrm{~mm}$ in 1st hr; Hb-13.7gm\%; USG- Normal scan; Peripheral smearUnremarkable. Skin biopsy: Findings were consistent with acute neutrophilic dermatosis (Figures 1 \& 2).

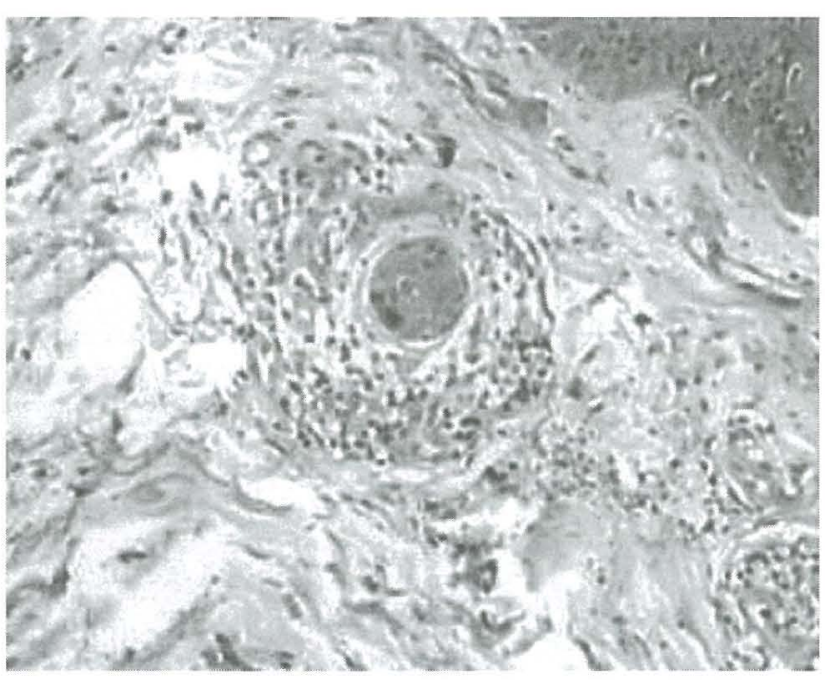

Fig.1. Skin biopsy showing inflammatory cells surrounding the blood vessels along with dermal oedema (HE stain) 


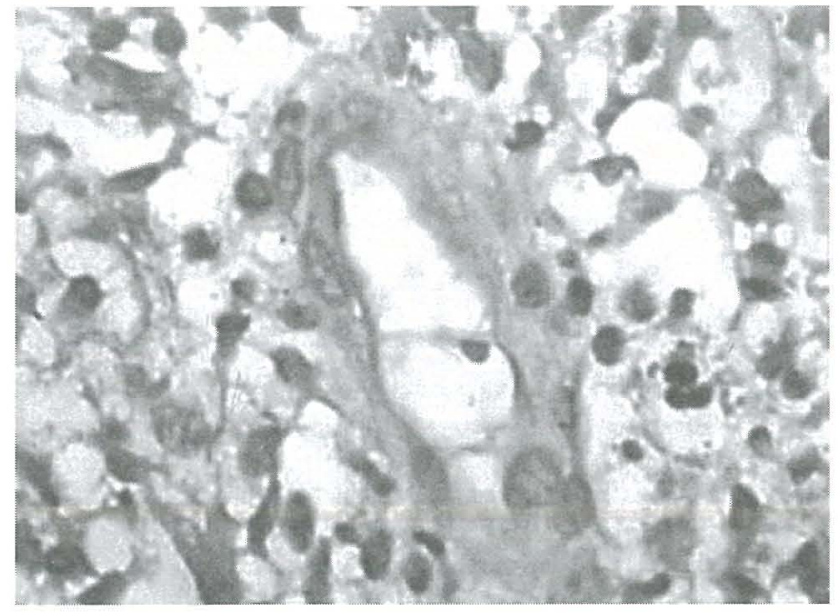

Fig. 2. Close up view showing intense neutrophil infiltrate in the upper dermis .

On the basis of history, typical skin lesions and investigations it was diagnosed as a case of Sweet's syndrome. Thus our case fulfilled both major and 3 of 4 minor criteria.

The patient was put on Tab. Prednisolone at the dose of 40mg daily along with Tab. Cetirizine and local application of Clobetasol cream.. Within 7 days the patient showed dramatic response and was free from all the symptoms and signs in less than a month. Its extremely good response to oral steroid was also an added point in favour of the diagnosis.

\section{Discussion}

Acute febrile neutrophilic dermatosis, first described in 1964 by Robert Douglas Sweet, has been termed Sweet's syndrome. ${ }^{1}$ The syndrome is characterized by pyrexia, elevated neutrophil count, painful red papules, nodules, plaques (which may be recurrent) and an infiltrate consisting predominantly of mature neutrophils that are diffusely distributed in the upper dermis. ${ }^{2}$ In addition to skin and mucosal lesions, Sweet's syndrome can also present with extra-cutaneous manifestations. Involvement of the eyes, joints, and oral mucosa as well as internal manifestations of Sweet's syndrome in the lung, liver, kidneys, heart, pancreas and central nervous system has been described. ${ }^{3}$ Diseases associated with Sweet syndrome include inflammatory bowel disease, and infections of the upper respiratory tract (e.g., streptococcosis) and the gastrointestinal tract (e.g., yersiniosis). ${ }^{4}$ There is a broad incidence peak in the fourth to seventh decades; women average age 50 and men age 65 . Disease onset clusters in the spring and autumn. The etiology is unknown but is thought to be a hypersensitivity reaction that leads to cytokine stimulation and subsequent neutrophil activation and infiltration. ${ }^{5}$ Sweet syndrome presents in three clinical settings: a classic or idiopathic form; a malignancyassociated or paraneoplastic form; and a druginduced form. Classic Sweet's syndrome occurs in middle-aged women after a nonspecific infection of the respiratory or gastrointestinal tract. Parainflammatory (e.g., infections, autoimmune disorders, vaccination) and paraneoplastic occurrence is found in approximately $25 \%$ of the cases and $2 \%$ are associated with pregnancy. Martinez et al. (2006) have reported the case of a patient who subsequently developed inflammatory dermatophytosis and Sweet's syndrome; an association not previously described. ${ }^{6}$ Associated malignant conditions are usually hematologic, most commonly acute myelogenous leukemia. Carcinomas of the breast and of the genitourinary and gastrointestinal tracts are the most frequently reported solid tumors. ${ }^{7}$ The skin lesion of sweet syndrome may be the initial manifestation of malignancy or may preceed the diagnosis by many months to years. Thus a close follow-up and screening is essential.

Medications implicated in drug-induced Sweet syndrome include granulocyte-colony stimulating factor, minocycline, trimethoprimsulfamethoxazole, antiepileptics, antihypertensives, oral contraceptives, or retinoids (Cohen et al.2000). The differential diagnosis for Sweet syndrome includes bowel bypass-related dermatosis, cellulitis or erysipelas, erythema nodosum, erythema multiforme, urticaria, necrotizing vasculitis, erythema elevatum diutinum, and pyoderma gangrenosum.

The most prominent histologic findings include a dense infiltrate of neutrophils in the superficial dermis and papillary dermal edema. Mononuclear cells and occasional eosinophils may be present. Additional findings may include leukocytoclasis and vesicles or bullae

Table 1.The diagnostic criteria of Sweet's syndrome (adapted from Cohen PR et al., 2000)

\begin{tabular}{|c|c|c|}
\hline Criteria & Classic/Malignancy-associated & Drug-induced \\
\hline 1 & \multicolumn{2}{|c|}{ Abrupt onset of painful erythematous plaques/nodules } \\
\hline 2 & \multicolumn{2}{|c|}{$\begin{array}{l}\text { Histopathologic evidence of a dense neutrophilic infiltrate without evidence of } \\
\text { leukocytoclastic vasculitis. }\end{array}$} \\
\hline 3 & \multicolumn{2}{|c|}{ Fever $>38^{\circ} \mathrm{C}$} \\
\hline 4 & $\begin{array}{l}\text { Association: underlying malignancy, } \\
\text { inflammatory disease, pregnancy; OR } \\
\text { preceded by upper respiratory or } \\
\text { gastrointestinal infection or vaccination. }\end{array}$ & $\begin{array}{l}\text { Temporal relationship: between } \\
\text { drug ingestion and clinical } \\
\text { presentation; OR recurrence after } \\
\text { oral challenge }\end{array}$ \\
\hline 5 & $\begin{array}{l}\text { Excellent response to treatment with } \\
\text { systemic glucocorticoids, or } \\
\text { Potassium iadide. }\end{array}$ & $\begin{array}{l}\text { Temporally related resolution of } \\
\text { lesions after drug withdrawal or } \\
\text { treatment with systemic } \\
\text { glucocorticoids. }\end{array}$ \\
\hline 6 & $\begin{array}{l}\text { Abnormal labs at presentation }(3 \text { of } 4 \text { ): } \\
\text { ESR }>20 \mathrm{~mm} / \mathrm{hr},(+) C \text { reactive protein, } \\
\text { WBC }>8000 \text {, neutrophils }>70 \text { percent }\end{array}$ & \\
\hline Diagnosis & $\begin{array}{l}\text { Both major criteria ( } 1 \text { and } 2) \text {, and } 2 \text { of } 4 \\
\text { minor criteria (3-6). }\end{array}$ & All 5 criteria \\
\hline
\end{tabular}


Sweet's syndrome responds rapidly to systemic therapy with corticosteroids and has been considered the 'gold standard' for the treatment of patients with Sweet's syndrome (Cohen and Kurzrock, 2002), but even after the treatment, it recurs in about $25 \%$ of the cases. ${ }^{8}$ Choi et al. have reported a case of recurrent Sweet's syndrome in an 80-year-old man with initial diagnosis of T cell pseudolymphoma. ${ }^{9}$ However, spontaneous resolution of the symptoms and lesions has occurred in several patients with Sweet's syndrome for whom disease-specific therapeutic intervention was not initiated and in some of the patients with drug-induced Sweet's syndrome after withdrawal of the dermatosis-causing medication. Alternative treatment modalities (e.g., doxycycline, potassium iodide, colchicine, indomethacin, dapsone, cyclosporine, cyclophosphamide, chlorambucil, and clofazamine, ) have also been used. Oral therapy with either potassium iodide or colchicine typically results in rapid resolution of Sweet's syndrome symptoms and lesions; therefore, in patients with Sweet's syndrome who have a potential systemic infection or in whom corticosteroids are contraindicated, it is reasonable to initiate treatment with these agents as a firstline therapy. Systemic antibacterials with activity against Staphylococcus aureus frequently result in partial improvement of Sweet's syndrome lesions when they are impetiginized or secondarily infected. In some patients with dermatosisassociated bacterial infections, organism-sensitive specific systemic antibacterials have been helpful in the management of their Sweet's syndrome. Although patients with hematologic malignancyassociated Sweet's syndrome often receive cytotoxic chemotherapy agents and antimetabolic drugs for the treatment of their underlying disorder, these agents are seldom used solely for the management of the symptoms and lesions of Sweet's syndrome. The treatment of patients with Sweet's syndrome with either etretinate or interferon-alpha has been reported as single case reports; both patients had improvement of not only their Sweet's syndrome lesions, but also their associated hematologic disorder. Localized lesions may be successfully treated with intralesional or topical glucocorticoids as either monotherapy or adjuvant therapy. ${ }^{10}$

\section{References:}

1. Von den Driesch P.Sweet's syndrome (acute febrile neutrophilic dermatosis). 1994 (4):53556.

2. Cohen PR, Kurzrock R. Sweet's syndrome: a review of current treatment options. 2002;3(2):117-31.

3. Vignon-Pennamen MD. The extracutaneous involvement in the neutrophilic dermatoses. Clin Dermatol. 2000; 18:339.

4. Joe EK.Sweet syndrome. Dermatol Online J 2003; 9 (4):28.

5. Cohen PR, et. al. Sweet's syndrome: A neutrophilic dermatosis classically associated with acute onset and fever. Clin Dermatol. 2000; 18:265.

6. Martinez W, del Pozo, J, PenaCMD; Yebra P, Maria $T$ et al. Sweet's syndrome in a woman with chronic dermatophytic infection. Int $\mathrm{J}$ Dermatol 2006; 45(11):1365-1368.

7. Hensley CD, et. al. Neutrophilic dermatoses associated with hematologic disorders. Clin Dermatol. 2000; 18:355.

8. Cohen PR. Sweet's syndrome--a comprehensive review of an acute febrile neutrophilic dermatosis. Orphanet J Rare Dis 2007;2:34.

9. Choi HJ, Chang SE, Lee MW, Choi JH, Moon $\mathrm{KC}$ et al. A case of recurrent Sweet's syndrome in an 80-year-old man: a clue to an underlying malignancy. Int J of Dermatol 2006;45(4):457459.

10. CohenP, KurzrockR. Sweet's syndrome revisited: a review of disease concepts. Int $\mathrm{J}$ Dermatol 2003;42(10): $\hat{E} 761-778$. 